\title{
Functional outcome after (laryngo)tracheal resection and reconstruction for acquired benign (laryngo)tracheal stenosis
}

\author{
Simone T. Timman ${ }^{1}$, Christiana Schoemaker ${ }^{1}$, Wilson W. L. Li ${ }^{1}$, Henri A. M. Marres ${ }^{2}$, Jimmie Honings ${ }^{2}$, \\ Wim J. Morshuis ${ }^{1}$, Erik H. F. M. van der Heijden ${ }^{3}$, Ad F. T. M. Verhagen ${ }^{1}$ \\ ${ }^{1}$ Department of Cardiothoracic Surgery, ${ }^{2}$ Department of Oto-Rhino-Laryngology and Head \& Neck Surgery, ${ }^{3}$ Department of Pulmonary Diseases, \\ Radboud University Medical Centre, Nijmegen, The Netherlands \\ Correspondence to: Simone T. Timman. Department of Cardiothoracic Surgery, Radboud University Medical Centre, Geert Grooteplein zuid 10 (route \\ 615), 6525 GA Nijmegen, The Netherlands. Email: simone.timman@radboudumc.nl.
}

\begin{abstract}
Background: In this study we focus on functional outcomes after (laryngo)tracheal resection and reconstruction for acquired benign (laryngo)tracheal stenosis, with a specific interest in the impact of laryngeal involvement on postoperative outcome.

Methods: All patients who underwent (laryngo)tracheal surgery for benign pathology between 1996 and 2017 in our centre were included in this retrospective study. Surgical outcomes were procedural success rate, and airway- and voice-related complications. Functional results were assessed using (standardized) questionnaires for quality of life, sensation of dyspnea, swallowing function, and voice perception.

Results: Of 119 consecutive patients, 47 underwent laryngotracheal resection and reconstruction and 72 underwent segmental tracheal surgery (78\% with an end-to-end tracheal anastomosis and $22 \%$ with a cricotracheal anastomosis). Overall success rate was $92 \%$ and was similar for all groups, with an overall significant improvement in quality of life when compared to the preoperative situation. However, after laryngotracheal surgery, airway-related complications were more common when compared to segmental resections with an end-to-end tracheal anastomosis (30\% versus $7 \%, \mathrm{P}=0.003)$. Additionally, early voice alterations without recurrent nerve palsy were reported twice as often ( $34 \%$ versus $16 \%, \mathrm{P}=0.034)$ and voice quality experienced during follow-up was significantly worse when compared to segmental resections. Overall response rate to the questionnaires on functional outcome was $63 \%$.

Conclusions: (Laryngo)tracheal surgery is safe and beneficial, with significant functional improvement during mid- and long-term follow-up. However, laryngeal involvement is a predictor for increased surgical airway-related complications. Additionally, voice alterations without recurrent nerve palsy are far more common after laryngotracheal resection and are a serious handicap. This aspect is underexposed in current literature and deserves further attention during preoperative counseling and patient follow-up. However, the results on functional outcome of this current study should be interpreted with caution due to the somewhat low response rate of the questionnaires.
\end{abstract}

Keywords: Laryngotracheal resection (LTR); tracheal surgery; long-term functional outcome

Submitted Dec 21, 2017. Accepted for publication Feb 27, 2018.

doi: 10.21037/acs.2018.03.07

View this article at: http://dx.doi.org/10.21037/acs.2018.03.07

\section{Introduction}

Acquired laryngotracheal stenosis (ALTS) and tracheal stenosis (ATS) are rare disorders caused by various diseases, both benign and malignant in nature, leading to gradually worsening dyspnea on exertion (1-3). Surgical resection has been advocated as the treatment of choice, with success rates reported from $84-100 \%$ after 1 month to 12 years of follow-up $(4,5)$. However, due to the complex anatomy when the subglottic or cricoid region is involved, a 
laryngotracheal resection (LTR) is more challenging when compared to an isolated segmental tracheal resection (STR), resulting in a less predictable outcome, with failure rates increasing to $37-70 \%(6,7)$.

While the above referenced studies were mainly focused on "surgical success" (i.e., recurrence of stenosis, successful decannulation, reintervention rates), various authors have advocated that functional outcome parameters, such as pulmonary-, voice-, and swallowing-related functions, are equally as important in defining treatment success (8). However, few studies have published these patient-reported functional outcomes after tracheal surgery in adults (3). Even when functional outcome parameters are being considered, only a minority of studies used standardized measurement tools to evaluate patient-reported outcome and quality of life (QoL) $(3,9)$.

In this study, we focus on functional outcomes after (laryngo)tracheal resection and reconstruction for acquired benign (laryngo)tracheal stenosis, with a specific emphasis on the impact of laryngeal involvement on postoperative results and functional outcomes.

\section{Methods}

\section{Patients and recruitment}

We performed a single centre retrospective cohort study, evaluating all patients who underwent (laryngo)tracheal resection for $\mathrm{A}(\mathrm{L}) \mathrm{TS}$ due to benign pathology in the Radboud University Medical Centre between January 1996 and December 2017. Carinal resections and procedures for congenital and/or malignant pathology were excluded from this analysis. The severity of preoperative stenosis was measured by computed tomography (CT). Percentage of stenosis was calculated by the cross-sectional area of the narrowest part of the stenosis divided by the surface of the trachea without obstruction. These percentages were categorised according the Cotton-Myer classification (Figure 1) (10). This study was approved by the local medical ethical reviewing committee.

Chart review was performed, collecting data on baseline patient characteristics, operative details, and surgical outcome parameters. Surgical outcome parameters ( $<6$ weeks after surgery) included: severe wound infection, haematoma, airway-related complications (e.g., restenosis, reintervention, dehiscence and respiratory insufficiency), voice-related complications [e.g., recurrent laryngeal nerve (RLN) palsy, voice alterations without recurrent nerve

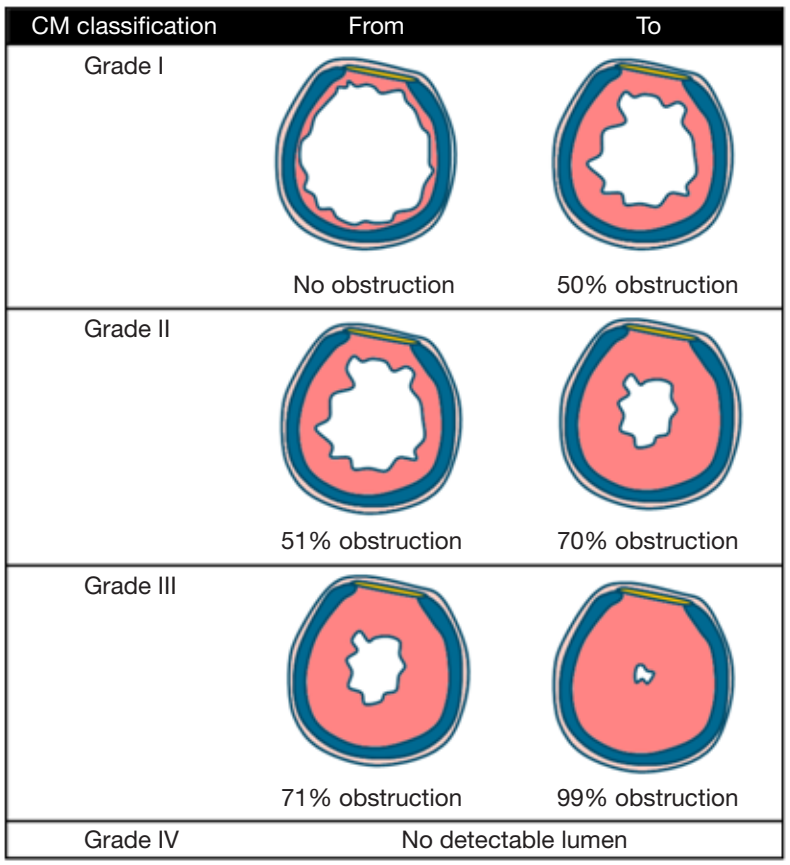

Figure 1 Severity of stenosis adapted from the Cotton-Myer classification (10).

palsy] and surgical mortality (30-day mortality and/or inhospital mortality). Postoperative success was defined as a sufficient airway ( $>90 \%$ rest lumen) without the need for reintervention up to 6 weeks after surgery.

Functional results were assessed by patient-reported functional outcome measures at least 6 months after surgery using (standardized) questionnaires, evaluating QoL, dyspnea- or swallowing related complaints and voice quality [voice handicap index (VHI)]. Questionnaires were distributed by mail in early 2016. Initial non-responders were sent the questionnaires again after 2 months or were contacted by telephone. In addition, starting in early 2017, functional outcome and QoL parameters were prospectively collected, including VHI, and data of eight additional patients were included for analysis.

\section{Functional results}

\section{QoL}

QoL was assessed by the EuroQol five dimensions questionnaire (EQ-5D), evaluating five aspects of perceived health problems, including mobility, self-care, usual activities, pain or discomfort, and anxiety or depression. We used the EuroQol visual analogue scale (EQ-VAS, 
0-10, 0 means the worst health imaginable and 10 means the best health imaginable) to record the patient's self-rated health state in the five different dimensions at the time of the questionnaire. A second VAS measurement was added for each dimension to score the preoperative situation as the patient could remember in order to retrospectively determine pre- and postoperative differences.

\section{Dyspnea}

Perception of dyspnea (in rest and during exercise), coughing, wheezing, inspiratory stridor and swallowing function were assessed by VAS with a similar two-line design as for the EQ-VAS to retrospectively compare the preoperative and current situation. The left end of the line was labelled "no complaints", versus "severe complaints" on the right end of the line.

\section{Pbonation}

Voice impairment was evaluated by the VHI. The VHI is a 30-item self-administered, validated questionnaire, designed to assess the impact of voice impairment in three different domains: functional, emotional and physical. A 4-point interval score from "never" (0 points) to "always" (4 points) was used to indicate the frequency of complaints (11). These scores can be combined to assign a total score, or they can be reflected separately into the three different domains, with higher scores indicating more severe voice impairment. In addition, a total of $1-30$ points is marked as a "mild dysfunction", 31-60 point as "moderate dysfunction" and above 60 points as "severe".

\section{Surgical procedure and postoperative care}

Patients were treated by a multidisciplinary team, which included the departments of cardiothoracic surgery, otolaryngology (head and neck surgery), and pulmonary diseases. (Laryngo)tracheal resections were performed by a similar technique as described by Grillo and Pearson et al. $(12,13)$. A cervical incision (with or without partial sternotomy) was performed for resections of the proximal and mid parts of the trachea. After dividing the isthmus of the thyroid, the anterior surface of the trachea was exposed and a circumferential dissection of the trachea was performed at the level of the diseased segment. STR was performed with an end-to-end tracheal anastomosis (STR-ETE) (Figure 2A) or, in case of resection of the first tracheal ring, a cricotracheal anastomosis (STRCT) was made (Figure 2B). For ALTS with involvement of the subglottic region, LTR was performed with two types of anastomosis. Firstly, after resection of the anterior cricoid, a thyro-crico-tracheal anastomosis (Figure 2C) was carried out. In five patients however, additional milling of the inner part of the cricoid was performed to gain extra lumen. The distal trachea was then directly attached to the thyroid cartilage anteriorly, but also to the mucosa of the thyroid on the lateral and dorsal side, forming a so-called thyrotracheal anastomosis. In all patients who underwent a LTR, the cricothyroid muscles were carefully detached to expose the anterior aspect of the larynx and reattached after completing the anastomosis to minimize voice alterations. All anastomoses were made using interrupted absorbable sutures $\left(\right.$ Vicryl $^{\circledR}$ 3.0). In case of a subglottic resection with an inlay of the membranous part, a running absorbable suture for the posterior membrane was used. A suprahyoid release (to reduce tension on the anastomosis) was avoided in all but one patient. A "guardian suture" or "chin suture" was routinely placed to avoid overextension of the neck and was removed after 7 days.

Intraoperative bronchoscopy was performed to assess the anastomosis and epiglottic swelling. A gastric feeding tube was placed intraoperatively. Most patients were extubated in the operation room and transferred to the intensive care unit or post anaesthesia care unit, although initially patients who had undergone a LTR remained intubated for a few days, until glottis and mucosal oedema had diminished. All patients received antibiotics for 5 days. Patients were not treated with steroids routinely, unless indicated by the pulmonologist. A second bronchoscopy was performed to evaluate the anastomosis and vocal cord function before discharge. Patients were usually discharged on postoperative day 7 to 10 . A final bronchoscopic assessment was done between 6 weeks and 3 months after surgery.

\section{Statistical analysis}

Continuous variables are reported as mean \pm standard deviation, or as median and range. Categorical variables are reported as numbers and percentages. $\chi^{2}$ and fisher's exact test were used for comparing categorical variables between two groups. To compare pre- and post-operative continuous variables, a related-sample or paired $t$-test was used. For comparison of continuous variables between two different groups an independent $t$-test was used. In case of non-normal distribution, a Mann-Whitney test or Wilcoxon non-parametric test was used for independent samples or related samples respectively. A P value $<0.05$ was 

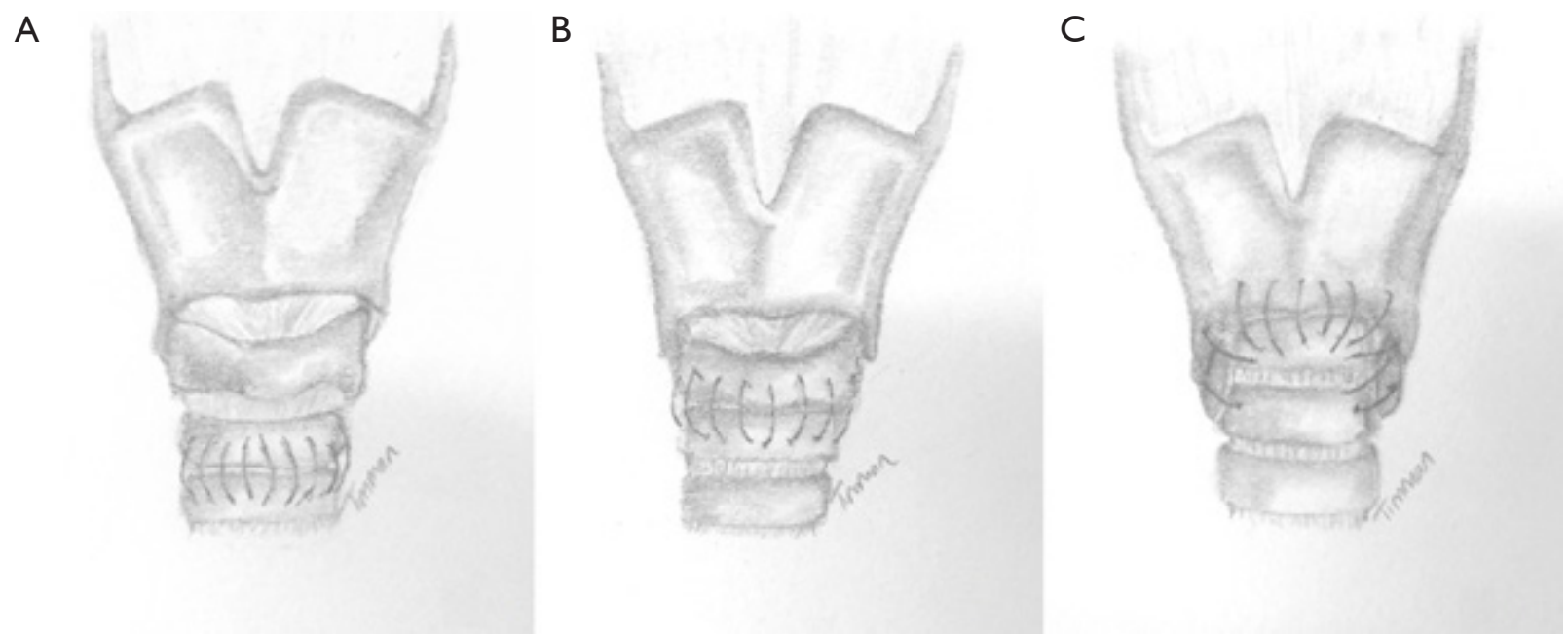

Figure 2 A tracheal end-to-end anastomosis (A) and a cricotracheal anastomosis (B) after a segmental tracheal resection, and a thyro-cricotracheal anastomosis $(\mathrm{C})$ after a laryngotracheal resection and reconstruction.

considered statistically significant. Statistical analysis was performed using Statistical Package for Social Sciences for Windows, version 22.0 (SPSS Inc., Chicago, Ill., USA).

\section{Results}

During the study period, 119 consecutive patients underwent LTR ( $\mathrm{n}=47)$ or STR with an end-to-end anastomosis (STRETE) $(n=56)$ or a cricotracheal anastomosis (STR-CT) $(n=16)$ in our centre (Table 1). Median age was 49 years (range, $0-85$ years) and $55 \%$ were female. Post-intubation tracheal stenosis (PITS) was the most common indication in all groups (47-91\%), predominantly in the STR-ETE group (91\%). In contrast, idiopathic tracheal stenosis (which is normally located in the laryngotracheal region) and granulomatosis with polyangiitis (formerly known as Wegener syndrome) were only seen in the LTR and STRCT patients. The severity of airway obstruction was not different between the groups. The majority of patients had a preoperative stenosis grade II or III, according to the Cotton-Myer classification.

In the LTR group, a thyro-crico-tracheal anastomosis was most commonly performed (89\%) and a thyrotracheal anastomosis in the rest of the cases. In all but one patient, the operation was performed through a cervical incision only. In the STR-ETE group, 91\% of patients were operated on through a cervical incision, with (partial) sternotomy being performed in 5\% of the cases. Resected segments were significantly longer in both STR groups compared to the LTR group (mean number of resected rings was 3 versus $5, \mathrm{P}<0.001)$. Six patients $(11 \%)$ in the STR-ETE and two patients $(4 \%)$ in the LTR group underwent long segmental $(>4.0 \mathrm{~cm})$ resection $(\mathrm{P}=0.285)$.

\section{Surgical outcome}

Surgical mortality was 1 out of $119(0.8 \%)$. This patient with Myhre syndrome underwent LTR and died inhospital after 4 months of stay on the ICU. Airway-related complications were observed in 14 patients after LTR $(30 \%)$ and in four patients after STR-ETE $(7 \%, \mathrm{P}=0.003)$ (Table 2). Respiratory insufficiency requiring reintubation (e.g., due to glottic oedema, pneumothorax) was seen in 6\% after LTR, versus $2 \%$ after STR $(\mathrm{P}=0.328)$. Restenosis rate was comparable after LTR (5 out of 47 patients, $11 \%$ ) and STR-ETE (4 out of 56, 7\%, P=0.490). No patients after STR-CT experienced restenosis. Of the nine patients in total with a restenosis, six patients (4 in the LTR group and 2 in the STR group) underwent endobronchial laser therapy or dilatation; one patient in the LTR group received highdose prednisolone. In addition, one patient in the LTR group and two patients in the STR-ETE group received a definitive tracheostomy. Surgical success rate after 6 weeks was $92 \%$ for all patients combined, $89 \%$ after LTR and 93\% after STR-ETE $(\mathrm{P}=0.729)$.

Regarding voice-related outcome, comparable rate of RLN palsy was found after LTR and STR-ETE ( $9 \%$ versus $4 \%, \mathrm{P}=0.408)$. However, early voice alterations without 


\begin{tabular}{|c|c|c|c|c|c|c|}
\hline Characteristics & $\begin{array}{l}\text { LTR } \\
(\mathrm{n}=47)\end{array}$ & $\begin{array}{l}\text { STR-CT } \\
(n=16)\end{array}$ & $\begin{array}{l}\text { STR-ETE } \\
(n=56)\end{array}$ & $\begin{array}{l}\mathrm{P} \text { value (LTR } \\
\text { vs. STR-ETE) }\end{array}$ & $\begin{array}{l}P \text { value (LTR } \\
\text { vs. STR-CT) }\end{array}$ & $\begin{array}{l}P \text { value (STR-CT } \\
\text { vs. STR-ETE) }\end{array}$ \\
\hline Age (years) & $43[0-85]$ & 50 [13-72] & $54[0-82]$ & 0.030 & 0.373 & 0.369 \\
\hline Gender (female) & $31[66]$ & $7[44]$ & $28[50]$ & 0.103 & 0.117 & 0.659 \\
\hline $\mathrm{BMI}\left(\mathrm{kg} / \mathrm{m}^{2}\right)$ & 27 [16-39] & $27[17-51]$ & 28 [14-48] & 0.406 & 0.842 & 0.659 \\
\hline Diabetes mellitus & $1[2]$ & $1[6]$ & $10[18]$ & 0.010 & 0.446 & 0.436 \\
\hline Connective tissue diseases & $6[13]$ & $2[13]$ & $2[4]$ & 0.138 & 1.000 & 0.212 \\
\hline \multicolumn{7}{|l|}{ Pathology } \\
\hline Postintubation stenosis & $22[47]$ & $11[69]$ & $51[91]$ & $<0.001$ & 0.528 & $<0.001$ \\
\hline Idiopathic tracheal stenosis & 15 [32] & $3[19]$ & - & - & - & - \\
\hline GPA/autoimmune related & $6[13]$ & $2[13]$ & - & - & - & - \\
\hline Grade I & $7[15]$ & 3 [19] & $8[14]$ & 0.268 & 0.567 & 0.914 \\
\hline Grade II & 9 [19] & $5[31]$ & 18 [32] & - & - & - \\
\hline Grade III & $14[30]$ & $5[31]$ & $20[36]$ & - & - & - \\
\hline Grade IV & $4[9]$ & - & 1 [2] & - & - & - \\
\hline Missing & $13[28]$ & $3[19]$ & $9[16]$ & - & - & - \\
\hline \multicolumn{7}{|l|}{ Previous intervention } \\
\hline Any type of intervention & $31[66]$ & $8[50]$ & $23[41]$ & 0.012 & 0.256 & 0.525 \\
\hline Dilatation & 23 [49] & $5[31]$ & $12[21]$ & - & - & - \\
\hline Laser & $12[26]$ & $5[31]$ & 7 [13] & - & - & - \\
\hline Type of anastomosis & & & & $\mathrm{N} / \mathrm{A}$ & $\mathrm{N} / \mathrm{A}$ & $\mathrm{N} / \mathrm{A}$ \\
\hline Thyro-tracheal & $5[11]$ & - & - & & & \\
\hline Thyro-crico-tracheal & $42[89]$ & - & - & & & \\
\hline Cricotracheal & - & 16 [100] & - & & & \\
\hline End-to-end tracheal & - & - & $56[100]$ & & & \\
\hline \multicolumn{7}{|l|}{ Type of incision } \\
\hline Cervical & $46[98]$ & $16[100]$ & $51[91]$ & 0.234 & 1.000 & 0.464 \\
\hline Cervical + (partial) sternotomy & - & - & $3[5]$ & - & - & - \\
\hline Sternotomy & 1 [2] & - & $2[4]$ & - & - & - \\
\hline \multicolumn{7}{|l|}{ Resected length } \\
\hline Number of rings & $3[1-8]$ & $5[2-7]$ & $5[1-9]$ & $<0.001$ & $<0.001$ & 0.559 \\
\hline Amount of $\mathrm{cm}$ & $2[1-4]$ & $3[1-4]$ & $3[1-5]$ & 0.006 & 0.063 & 0.765 \\
\hline Long segment $(>4.0 \mathrm{~cm})$ & $2[4]$ & - & $6[11]$ & 0.285 & 1.000 & 0.327 \\
\hline Operation time (minutes) & 165 [118-385] & $165[120-347]$ & $150[97-279]$ & 0.041 & 0.807 & 0.239 \\
\hline
\end{tabular}

Continuous variables are expressed as median (range); dichotomous variables are expressed as count (\%). LTR, laryngotracheal resection; STR, segmental tracheal resection; BMI, body mass index; TEF, transesophageal fistula; STR-CT, STR with a cricotracheal anastomosis; STR-ETE, STR with an end-to-end tracheal anastomosis; COPD, chronic obstructive pulmonary disease; CM, Cotton-Myer classification; N/A, not available; GPA, granulomatosis with polyangiitis. 


\begin{tabular}{|c|c|c|c|c|c|c|}
\hline Extubation $>24 \mathrm{~h}$ & $17[36]$ & $3[19]$ & $6[11]$ & 0.001 & 0.445 & 0.281 \\
\hline \multicolumn{7}{|l|}{ Airway-related complications } \\
\hline Respiratory insufficiency ${ }^{\infty}$ & $3[6]$ & $2[13]$ & $1[2]$ & 0.328 & 0.597 & 0.125 \\
\hline Pulmonary infection & 4 [9] & $2[13]$ & $2[4]$ & 0.407 & 0.643 & 0.217 \\
\hline Oedema & $10[21]$ & $3[19]$ & - & $<0.001$ & 1.000 & 0.100 \\
\hline Postoperative cannula & - & - & - & - & - & - \\
\hline \multicolumn{7}{|l|}{ Voice-related complications } \\
\hline Total & 20 [43] & $2[13]$ & $11[20]$ & $<0.001$ & 0.012 & 0.722 \\
\hline RLN palsy & 4 [9] & - & $2[4]$ & 0.408 & 0.546 & 1.000 \\
\hline Voice alterations without RLN palsy & 16 [34] & $2[13]$ & $9[16]$ & 0.034 & 0.121 & 1.000 \\
\hline Dysphagia & 4 [9] & $1[6]$ & $6[11]$ & 0.752 & 1.000 & 1.000 \\
\hline \multicolumn{7}{|l|}{ Surgical mortality } \\
\hline
\end{tabular}

RLN palsy were significantly greater after LTR when compared to STR-ETE (34\% versus $16 \%, \mathrm{P}=0.034$ ).

\section{Functional outcome}

Of the total of 119 patients, 75 patients had more than 6 months follow-up and were alive at the time of the distribution of the questionnaires. Of these, 47 patients (63\%) responded to the questionnaires, 17 patients (36\%) in the LTR and 8 (17\%) and $22(47 \%)$ patients in de STRCT and STR-ETE groups respectively. Mean follow-up at the time of the questionnaire was comparable between all groups (mean, 5.3-5.8 years; range, 0.55-14.70 years). In addition, after the initiation of the prospective database,
VHI questionnaires from an additional eight patients were collected (seven patients after LTR, one after STR-ETE) and included in this analysis, totaling 55 patients providing VHI data.

With regard to QoL (Table 3), there was a significant improvement in 2 out of 5 domains of the EQ5D-VAS after LTR and STR-CT, and in four domains after STRETE when compared to the preoperative situation. There were no significant differences among the three groups. All airway-related scores improved significantly in the LTR and STR-ETE groups, with the most substantial improvement for dyspnea during exercise $(+5.6$ versus +3.8 points for LTR and STR respectively, $\mathrm{P}=0.239$ ) and inspiratory stridor $(+5.8$ versus +4.4 points, $\mathrm{P}=0.283)$. We 


\begin{tabular}{|c|c|c|c|c|c|c|}
\hline Duration of follow-up & $5.5( \pm 3.9)$ & $5.8( \pm 3.7)$ & $5.3( \pm 3.6)$ & 0.871 & 0.892 & 0.794 \\
\hline \multicolumn{7}{|l|}{ EQ5D-VAS (0-10 points) ${ }^{*}$} \\
\hline Self-care & $+1.4( \pm 3.1)$ & $+1.6( \pm 4.1)$ & $+2.1( \pm 3.9)^{\#}$ & 0.672 & 0.626 & 0.360 \\
\hline Usual activities & $+2.7( \pm 4.5)^{\#}$ & $+3.8( \pm 3.7)^{\#}$ & $+2.3( \pm 3.8)^{\#}$ & 0.599 & 0.599 & 0.269 \\
\hline Pain or discomfort & $+1.9( \pm 5.0)$ & $+4.6( \pm 3.5)^{\#}$ & $+1.7( \pm 4.0)^{\#}$ & 0.669 & 0.208 & 0.076 \\
\hline Anxiety or depression & $+2.4( \pm 2.5)^{\#}$ & $+0.5( \pm 2.7)$ & $+1.3( \pm 3.3)$ & 0.506 & 0.088 & 0.299 \\
\hline Dyspnea during exercise & $+5.6( \pm 3.8)^{\#}$ & $+5.2( \pm 5.4)^{\#}$ & $+3.8( \pm 4.8)^{\#}$ & 0.239 & 0.930 & 0.396 \\
\hline Wheezing & $+2.9( \pm 4.1)^{\#}$ & $+2.7( \pm 4.5)$ & $+3.9( \pm 4.8)^{\#}$ & 0.557 & 0.229 & 0.421 \\
\hline Inspiratory stridor & $+5.8( \pm 3.5)^{\#}$ & $+4.3( \pm 4.2)^{\#}$ & $+4.4( \pm 5.0)^{\#}$ & 0.283 & 0.380 & 0.866 \\
\hline Coughing & $+3.8( \pm 3.6)^{\#}$ & $+2.6( \pm 4.8)$ & $+1.6( \pm 4.1)^{\#}$ & 0.147 & 0.599 & 0.708 \\
\hline \multicolumn{7}{|c|}{ Swallowing-related outcome (0-10 points) ${ }^{\star}$} \\
\hline Dysphagia score & $+0.1( \pm 2.0)$ & $+2.1( \pm 4.7)$ & $+1.7( \pm 4.8)$ & 0.303 & 0.844 & 0.922 \\
\hline \multicolumn{7}{|l|}{ Voice-related outcome (VHI) } \\
\hline Mild & 13 [52] & $5[6]$ & $11[48]$ & 0.662 & 0.216 & 0.435 \\
\hline Moderate & $5[20]$ & - & 2 [9] & - & - & - \\
\hline Severe & $4[16]$ & - & 2 [9] & - & - & - \\
\hline Number of inclusion for the VHI & 25 & 8 & 23 & - & - & - \\
\hline
\end{tabular}

Variables are presented as mean \pm standard deviation of count (\%). * differences between postoperative evaluation and preoperative estimation according to visual analogue scale (VAS); +, indicates an improvement of symptoms; -, indicates worsening of symptoms; \#, significant differences between postoperative evaluation and preoperative estimation according to VAS; ${ }^{\wedge}$, higher score indicate more severe voice impairment. LTR, laryngotracheal resection; STR, segmental tracheal resection; EQ5D, EuroQol 5 dimensions questionnaire; $\mathrm{VHI}$, voice handicap index.

did not find significant swallowing complaints after surgery in our series.

In all groups, any voice alteration was reported after surgery. The mean total VHI score was higher (indicating more impairment) in the LTR group than in the STR-ETE group (28.6 versus 17.4 points, $\mathrm{P}=0.027$ ) (Table 3). Four patients $(16 \%)$ in the LTR and $2(9 \%)$ in the STR-ETE group had a score above 60 points, which is considered severe voice dysfunction. In total, seven patients had moderate voice dysfunction, 5 (20\%) in the LTR and 2 (9\%) 
in the STR-ETE group. Thirteen patients in the LTR group had a mild voice dysfunction $(\mathrm{P}=0.662)$.

\section{Discussion}

In our study of 119 patients, the overall success rate was high and was similar for patients after LTR (89\%) and STR-ETE (93\%). The main focus of this study was to assess the impact of laryngeal involvement on postoperative results and functional outcome. Therefore, the emphasis of comparison was mainly between the LTR and the STRETE group. The STR-CT group can be considered an "in between group", as we hypothesize that this is less invasive when compared to a LTR. During follow-up, the LTR and STR-ETE groups experienced a significant improvement in QoL and airway-related sensation scores. However, patients after LTR had significantly more early airway-related complications and experienced significantly more voice impairment without RLN palsy, both early postoperatively and during long-term follow-up (mean, 5.5-5.8 years).

The success rate reported in our series is consistent with other reports, varying between $83 \%$ and $98 \%$ after single-stage (laryngo)tracheal resection and reconstruction. Furthermore, (laryngo)tracheal surgery appears safe, with a mortality of $0.8 \%$ in our series, which is in accordance with a surgical mortality of $0 \%$ to $2 \%$ as reported in the literature (5,14-19).

Interestingly, we found significantly more airway-related complications after LTR versus STR, with a trend towards a higher risk of restenosis. This strengthens the notion that LTR is a more challenging and complex procedure with less predictable outcomes when compared to STR $(6,14,15)$. This is probably due to the anatomical challenges in the laryngeal zone, caused by a very narrow tracheal lumen in this region, the attachment of the vocal cords to the arytenoids, as well as the branches of the RLN which are attached to the lateral sides of the cricoid.

Tracheal surgery is primarily focused on restoring upper airway function. Most reports focus on post-procedural tracheal lumen patency or decannulation rates as the definition for treatment success $(5,7,14,15,18,20)$. However, functional outcome should be considered equally important, especially from the patients' point of view. Currently there is only limited data to understand the extent and impact of voice and swallowing difficulties in patients $(3,9,21)$. In our series, patients reported a consistent long-term improvement of dyspnea-, stridor- and coughing sensation scores in both major groups after a mean follow-up of
5.5 years. Similarly, Liberman and Mathisen (22) also reported remarkable improvement of dyspnea sensation, wheezing and coughing severity without impairment of swallowing after a tailored cricoplasty; however, their follow-up period was less than 2 years. To the best of our knowledge, our study presents functional data with the longest follow-up in the literature, confirming the longterm and lasting success of (laryngo)tracheal resection for various diseases.

Of special interest, voice function seems to be more significantly impaired after LTR compared to STR-ETE. The STR-CT group showed similar rates as in the STRETE group but due to small number of patients, the differences between the LTR and STR-CT group were not statistically significant. In our series, RLN palsy was seen in four patients (9\%) after LTR and in two patients (4\%) after STR-ETE. Our results are comparable with findings in other reported series, where RLN palsy is found in $2 \%$ to $13 \%$ of patients after (laryngo)tracheal surgery $(20,23,24)$. On the other hand, voice alterations without RLN palsy were reported twice as much after LTR when compared to STR-ETE (34\% versus $16 \%, \mathrm{P}=0.034$ ). This is similar to the study by Menapace $e t a l$. reporting $31 \%$ of poor voice outcome after LTR (25). Voice alteration without RLN palsy was noted in only two patients (13\%) after STR-CT, which corresponds with our hypothesis that a cricotracheal anastomosis (STR-CT) is less invasive when compared to LTR and subsequently has less impact on voice function. We speculate that voice impairment or alterations after LTR, without RLN palsy, are at least partially caused by a change in the muscle length of the cricothyroid muscle, which overlies the anterior cricoid cartilage. The reattachment of the cricothyroid muscle influences the functional belly length. When the muscle belly is relatively too long, this will result in a lower muscle tension, causing a deeper voice. From our point of view, voice function seems to be an overlooked aspect after tracheal surgery. Just five medium-sized to large case-series (including more than 50 patients) mentioned abnormal voice outcome without RLN palsy after tracheal surgery $(15-18,26)$. Only one study with 32 patients quantified voice outcome from good to poor (25). Patients, especially (young) female patients, can experience a deeper voice after surgery as a serious handicap $(9,27)$. As shown in our current study, voice dysfunction is indeed an existing and a relevant problem, especially after LTR. As such, it should be part of preoperative counseling and informed consent. In addition, patients' vocal demands should be taken into account in surgical decision making. 
Although our study reports the longest functional follow-up after (laryngo)tracheal surgery in a large group of patients, several limitations of the study affect the strength of our findings and conclusions. An important limitation of this study is our retrospective baseline measurement for QoL. Recall bias is present to a large extent. Nevertheless, we believe that patients are able to mark any improvement or worsening after surgery retrospectively. Furthermore, the response rate for the functional measurements was low $(63 \%)$, despite sending multiple questionnaires. This may be caused by the time frame of our study, as some patients have had surgery 20 years ago. This non-response bias may have significantly impacted our findings and conclusions, with the possibility that patients' outcomes (whether positive or negative) affected their likelihood of responding to our questionnaires.

To address the before mentioned limitations, we have initiated a project to evaluate all patients undergoing tracheal surgery in our centre in a prospective matter, with preoperative baseline and postoperative QoL measurements through standardized questionnaires. In addition, we want to quantify voice quality by using phone tography.

In conclusion, (laryngo)tracheal resection and reconstruction is safe with low surgical mortality and a high success rate. We found a consistent functional improvement during follow-up, especially in dyspnea sensation during exercise and reduction of inspiratory stridor in all groups, without impaired swallowing. However, after resections and reconstructions involving the laryngeal region, airwayrelated complications were significantly more common. In addition, voice impairment (without RLN palsy) is found more frequently (34\%) when compared to STR-ETE (16\%). A significant higher VHI score after LTR also points out that patients do perceive their voice alteration as a serious handicap. Therefore, these findings should be used to more adequately inform patients preoperatively. However, the reports on functional outcome of this current study should be interpreted with caution due to the somewhat low response rate of the questionnaires $(63 \%)$.

\section{Acknowledgements}

None.

\section{Footnote}

Conflicts of Interest: The abstract of this manuscript was presented at the 31st EACTS Annual Meeting in Vienna,
Oct 10, 2017.

\section{References}

1. Grillo HC. Primary reconstruction of airway after resection of subglottic laryngeal and upper tracheal stenosis. Ann Thorac Surg 1982;33:3-18.

2. Lewis S, Earley M, Rosenfeld R, et al. Systematic review for surgical treatment of adult and adolescent laryngotracheal stenosis. Laryngoscope 2017;127:191-8.

3. Clunie GM, Kinshuck AJ, Sandhu GS, et al. Voice and swallowing outcomes for adults undergoing reconstructive surgery for laryngotracheal stenosis. Curr Opin Otolaryngol Head Neck Surg 2017;25:195-9.

4. Yamamoto K, Kojima F, Tomiyama K, et al. Meta-analysis of therapeutic procedures for acquired subglottic stenosis in adults. Ann Thorac Surg 2011;91:1747-53.

5. Wright CD, Grillo HC, Wain JC, et al. Anastomotic complications after tracheal resection: prognostic factors and management. J Thorac Cardiovasc Surg 2004;128:731-9.

6. Abbasidezfouli A, Akbarian E, Shadmehr MB, et al. The etiological factors of recurrence after tracheal resection and reconstruction in post-intubation stenosis. Interact Cardiovasc Thorac Surg 2009;9:446-9.

7. Abouyared M, Szczupak M, Barbarite E, et al. Open airway reconstruction in adults: Outcomes and prognostic factors. Am J Otolaryngol 2017;38:7-12.

8. van den Boogert J, Hans Hoeve LJ, Struijs A, et al. Singlestage surgical repair of benign laryngotracheal stenosis in adults. Head Neck 2004;26:111-7.

9. Bryans L, Palmer AD, Schindler JS, et al. Subjective and objective parameters of the adult female voice after cricotracheal resection and dilation. Ann Otol Rhinol Laryngol 2013;122:707-16.

10. Myer CM 3rd, O'Connor DM, Cotton RT. Proposed grading system for subglottic stenosis based on endotracheal tube sizes. Ann Otol Rhinol Laryngol 1994;103:319-23.

11. Jacobson BH, Johnson A, Grywalski C, et al. The Voice Handicap Index (VHI): Development and Validation. Am J Speech Lang Pathol 1997;6:66-70.

12. Grillo HC. The management of tracheal stenosis following assisted respiration. J Thorac Cardiovasc Surg 1969;57:52-71.

13. Pearson FG, Cooper JD, Nelems JM, et al. Primary tracheal anastomosis after resection of the cricoid cartilage with preservation of recurrent laryngeal nerves. J Thorac 
Cardiovasc Surg 1975;70:806-16.

14. Grillo HC, Mathisen DJ, Wain JC. Laryngotracheal resection and reconstruction for subglottic stenosis. Ann Thorac Surg 1992;53:54-63.

15. Bibas BJ, Terra RM, Oliveira Junior AL, et al. Predictors for postoperative complications after tracheal resection. Ann Thorac Surg 2014;98:277-82.

16. D'Andrilli A, Maurizi G, Andreetti C, et al. Longterm results of laryngotracheal resection for benign stenosis from a series of 109 consecutive patients. Eur J Cardiothorac Surg 2016;50:105-9.

17. Herrington HC, Weber SM, Andersen PE. Modern management of laryngotracheal stenosis. Laryngoscope 2006;116:1553-7.

18. Rea F, Callegaro D, Loy M, et al. Benign tracheal and laryngotracheal stenosis: surgical treatment and results. Eur J Cardiothorac Surg 2002;22:352-6.

19. Amorós JM, Ramos R, Villalonga R, et al. Tracheal and cricotracheal resection for laryngotracheal stenosis: experience in 54 consecutive cases. Eur J Cardiothorac Surg 2006;29:35-9.

20. Rubikas R, Matukaityte I, Jelisiejevas JJ, et al. Surgical treatment of non-malignant laryngotracheal stenosis. Eur Arch Otorhinolaryngol 2014;271:2481-7.

21. Lennon CJ, Gelbard A, Bartow C, et al. Dysphagia

Cite this article as: Timman ST, Schoemaker C, Li WW, Marres HA, Honings J, Morshuis WJ, van der Heijden EH, Verhagen AF. Functional outcome after (laryngo)tracheal resection and reconstruction for acquired benign (laryngo) tracheal stenosis. Ann Cardiothorac Surg 2018;7(2):227-236. doi: 10.21037/acs.2018.03.07
Following Airway Reconstruction in Adults. JAMA Otolaryngol Head Neck Surg 2016;142:20-4.

22. Liberman M, Mathisen DJ. Tailored cricoplasty: an improved modification for reconstruction in subglottic tracheal stenosis. J Thorac Cardiovasc Surg 2009;137:5738; discussion 578-9.

23. El-Fattah AM, Ebada HA, Amer HE, et al. Partial cricotracheal resection for severe upper tracheal stenosis: Potential impacts on the outcome. Auris Nasus Larynx 2018;45:116-22.

24. Piazza C, Del Bon F, Paderno A, et al. Complications after tracheal and cricotracheal resection and anastomosis for inflammatory and neoplastic stenoses. Ann Otol Rhinol Laryngol 2014;123:798-804.

25. Menapace DC, Modest MC, Ekbom DC, et al. Idiopathic Subglottic Stenosis: Long-Term Outcomes of Open Surgical Techniques. Otolaryngol Head Neck Surg 2017;156:906-11.

26. Macchiarini P, Verhoye JP, Chapelier A, et al. Partial cricoidectomy with primary thyrotracheal anastomosis for postintubation subglottic stenosis. J Thorac Cardiovasc Surg 2001;121:68-76.

27. Smith ME, Roy N, Stoddard K, et al. How does cricotracheal resection affect the female voice? Ann Otol Rhinol Laryngol 2008;117:85-9. 